\title{
Folate Deficiency and Its Impact on NTDs Prevalence in Georgia
}

\author{
Robizon Tsiklauri ${ }^{1 *}$, I Parvanta ${ }^{2}$, L Jijeishvili ${ }^{3}$, M Kherkheulidze $^{4}$ and N Kazakhashvili ${ }^{5}$ \\ ${ }^{1}$ National Center for Disease Control and Public Health Georgia \\ ${ }^{2}$ International expert \\ ${ }^{3}$ LTD Vistamedi \\ ${ }^{4}$ Medical University \\ ${ }^{5} U G$
}

*Corresponding author: Robizon Tsiklauri, National Center for Disease Control and Public Health 99, Kakheti highway; 0198

Tbilisi, Georgia

\section{ARTICLE INFO}

Received: 幽 November 14, 2019

Published: 㓞 November 20, 2019

Citation: Robizon Tsiklauri, I Parvanta, L Jijeishvili, M Kherkheulidz, N Kazakhashvili. Folate Deficiency and Its Impact on NTDs Prevalence in Georgia. Biomed J Sci \& Tech Res 23(1)-2019. BJSTR. MS.ID.003853.

Abbreviations: IRB: Institutional Review Board, NCDC: National Centre for Disease Control, NTDs: Neural Tube Defects, WHO: World Health Organization.

\section{ABSTRACT}

Background: Until 2015, systematic statistical data on micronutrient deficiency was not available in Georgia, to provide developing national strategy. In the same year, the National Centre for Disease Control and Public Health of Georgia (NCDC) in collaboration with the USA CDC launched the project "Strengthening surveillance of micronutrient deficiency in Georgia".

Design \& Methodology: In 2015 we did choose sentinel surveillance approach. For setting nutrition surveillance system 8 sentinel sites $(2$ sites in each region/children and pregnant health facilities) in four regions of Georgia were selected, using the criteria of geographical, social, ethnical, urban/rural, and religion. The project protocols were approved by the Institutional review board (IRB) at the NCDC and by the Research Review Committee and Ethical review committee of the US CDC.

Results: As a result of surveillance system functioning (2016-2019) we reviled that, about $12.4 \%$ among tested 3030 pregnant women on $\mathrm{Hb}$ were found anemic. Additionally, 963 were tested for iron and folate deficiencies. $57 \%$ were iron deficient, and $28 \%$ tested positive for folate deficiency. Neural tube defects (NTDs) prevalence per 1000 live births registered in sentinel sites was high 3.7.

Conclusion: Our results show that anemia and iron deficiency are prevalent among both pregnant women and children of the specified age group in Georgia. Additionally, folate deficiency was quite common during the1st trimester of pregnancy. Our findings will inform public health policy decision makers to take relevant decisions on required interventions, such as health education, distribution of relevant supplements, and food fortification.

\section{Introduction}

Micronutrient deficiencies form an important global health issue, with malnutrition affecting key development outcomes including poor physical and mental development in children, vulnerability or exacerbation of disease, mental retardation, blindness and general losses in productivity and potential Unlike energy-protein undernourishment, the health impacts of micronutrient deficiency are not always acutely visible; it is therefore sometimes termed 'hidden hunger' (the two terms can be used interchangeably). The World Health Organization (WHO) estimate that more than 2 billion people suffer from micronutrient deficiency globally [1]. Epidemiological studies for assessing the micronutrient status among the population in the South Caucasus 
region which includes Georgia, Azerbaijan, and Armenia, are limited. Reports from Armenia (2000) estimated the prevalence of anemia to be $12 \%$ and $12.4 \%$ among pregnant women and nonpregnant women, respectively and to be $23.9 \%$ in children under five [2].

A nationwide survey (2009) in Georgia reported prevalence of $22.8 \%$ anemic children, $25.6 \%$ in pregnant and $36.6 \%$ in non-pregnant women [3]. Iron is an essential element for the biosynthesis of blood hemoglobin. The symptoms of iron deficiency anemia can be mild at first and are not diagnosed until they have a routine blood test. Potential health complications of iron deficiency anemia include rapid or irregular heartbeat, pregnancy complications of premature birth or low birth weight, and delayed growth in infants and children [4]. Folate insufficiency manifests in neural tube defects (NTDs), which is caused by low concentration of vitamin B9(folate) in blood. Approximately 190,000 neonates are born with NTD in low Income Countries. TDs are serious and most common consequence of folic acid deficiencies. NTDs occur when neural tube closure is completed by embryonic day 28 of pregnancy and arise when the neural tube cannot close properly. The most common NTDs are the following: anencephaly and spina bifida [5].

Neural tube defects (NTDs), including spina bifida and anencephaly, are among the commonest human birth defects, affecting around 1 in every 1000 pregnancies. While the causes of human NTDs remain poorly understood in most cases, more than 100 single-gene defects can cause NTDs in mouse models. Elevated homocysteine concentration in maternal blood is a risk factor for human NTDs and was found to occur in association with reduced maternal and embryonic folate in our dietary models. A prevalent idea is that homocysteine itself may cause NTDs [6]. Nutritional deficiencies are preventable etiological and epigenetic factors causing congenital abnormalities, first cause of infant mortality. Folate deficiency has a well-established teratogenic effect, leading to an increasing risk of neural tube defects. This paper highlights the most recent medical literature about folate deficiency, be it maternal or paternal. It then focuses on associated deficiencies as nutritional deficiencies are multiple and interrelated. Observational and interventional studies have all been consistent with a $50-70 \%$ protective effect of adequate women consumption of folates on neural tube defects. Since strategies to modify women's dietary habits and vitamin use have achieved little progress, scientific as well as political effort is mandatory in order to implement global preventive public health strategies aimed at improving the alimentation of women in reproductive age, especially folic acid supplementation. Even with the recent breakthrough of fetal surgery for myelomeningocele, the emphasis should still be on prevention as the best practice rather than treatment of neural tube defects [7].

NTDs are the second most common form of severe congenital defects after heart defects, afflicting approximately 300000 infants across the world annually. The prevalence of NTDs ranges from 5-60 per 10000 births, which is most likely since the prevalence data originate from different populations, ethnicities and regions. Maternal health and nutritional status can modulate the development of neural tube. In this regard, folic acid (FA) intake has had a significant impact on reducing the overall incidence of NTDs. However, this approach has not provided the complete elimination of NTDs. Thus, additional modifiable factors, such as vitamin B12, may play roles in the prevention of NTDs, as folate together with vitamin B12 conduct the transfer of single carbon units and methylation reactions needed for the synthesis of nucleotides in cells [8].

Despite efforts to tackle folate deficiency and Neural Tube Defects (NTDs) through folic acid fortification, its implementation is still lacking where it is needed most, highlighting the need for studies that evaluate the effectiveness of folic acid fortified wheat flour in a poor, rural, high-risk, NTD region of China. One of the most affected regions, Shanxi Province, was selected as a case study. A community intervention was carried out in which 16,648 women of child-bearing age received fortified flour (eight villages) and a control group received ordinary flour (three villages). NTD birth prevalence and biological indicators were measured two years after program initiation at end line only. The effect on the NTD burden was calculated using the disability-adjusted life years (DALYs) method. In the intervention group, serum folate level was higher than in the control group. NTDs in the intervention group were $68.2 \%$ lower than in the control group. In terms of DALYs, burden in intervention group was approximately $58.5 \%$ lower than in the control group. Flour fortification was associated with lower birth prevalence and burden of NTDs in economically developing regions with a high risk of NTDs [9].

Folate deficiency in the periconceptional period contributes to neural tube defects; deficits in vitamin B12 (cobalamin) have negative consequences on the developing brain during infancy; and deficits of both vitamins are associated with a greater risk of depression during adulthood. This review examines two mechanisms linking folate and vitamin B12 deficiency to abnormal behavior and development in infants: disruptions to myelination and inflammatory processes. Future investigations should focus on the relationship between the timing of deficient and marginal vitamin B12 status and outcomes such as infant growth, cognition, social development, and depressive symptoms, along with prevention of folate and vitamin B12 deficiency [10].

\section{Potential Target Group Sand Study Design}

Potential target groups for surveillance of micronutrient deficiency are infants, toddlers, preschool children, school-age children, and women of childbearing age. Toddlers are vulnerable to micronutrient deficiencies, are accessible for the assessment in child health clinics and community surveys and are an indicator of risk in the general population. Surveillance of iron deficiency is 
focused on these vulnerable groups. In the present study women at the $1^{\text {st }}$ trimester of pregnancy was studied. The study focused on following population groups including toddlers (aged $12-23$ months old), and pregnant. Selection of these target groups were based on level of risk or vulnerability, accessibility of the target group for assessment, and degree of representativeness, or the ability to reflect the extent of the problem in the overall population. Other criteria included the availability of normative data and the potential usefulness of the targeted population for surveillance of other micronutrient deficiencies.

\section{Objectives of the study}

Studying of micronutrient deficiency prevalence in children and pregnant women.

\section{Project Implementation Stages}

Selecting the Sentinel Sites: Sentinel sites selection has been undertaken considering the main characteristics of representativeness (geographical, social, ethnical, urban/rural, religion, etc.) and existing information about malnutrition and dietary habits. All people living in selected region had an equal chance to participate in the study. 8 sentinel sites (health facilities) in 4 Regions of Georgia (Achara, Samegrelo, Tbilisi, Kakheti) were selected for this pilot project, 4 sites for gating nutritional status data from children and, 4 sites - from pregnant.

Selecting the Target Groups: Target groups from whom data were collected include:
a. Pregnant
b. Children U2 (12-23 months age)
c. School age children (12 years old)

Selecting the Nutritional Indicators: 5 nutritional indicators were selected for this project (Iron, Folate, Ca, vitamin "D", and Iodine).

Data Collection: Two types of data collection were used: extracting existing data from selected health facilities (sentinel sites): Hemoglobin Data and Anthropometric measurement data. For both types of data collection relevant 'Data Collection forms' were utilized. Second type of data collection - was coming from laboratory results of blood.

Trainings: Project team (manager and local consultants) conducted trainings for sentinels' staff on 3 main themes:

a. Nutrition status surveillance and monitoring specifics \& special program forms preparing (electronic form of data base) and submitting,

b. Rickets and vitamin "D" deficiency study, management and prevention, and

c. Lead poisoning, study and management.
Testing and Data Collection were Realized by the Following Study Scheme:

a. Hemoglobin /in pregnant $\left(1^{\text {st }}\right.$ trimester $)$ visiting sentinel site

b. Iron and folate deficiencies (laboratory testing) were studded in pregnant (1st trimester)

c. Hemoglobin /in children (12-23 months of age) visiting sentinel site, for immunization

d. Iron, Ca and vitamin "D" deficiencies (laboratory testing) in children 12-23 months of age.

e. Anthropometric measurement was used for children.

f. And, NTDs prevalence monitoring is also involved in surveillance system. Laboratory testing's on iron, folate, Ca and vitamin "D" deficiencies were conducted in Tbilisi reference Laboratory.

g. Iodine in PW and SAC.

Laboratory Methodology: Laboratory testing of blood samples was used in this study project/well-accepted.

a. After explaining the aim of the study (informal consent), a blood sample was collected from the anti-cubital vein in heparinized tube. Next, the sample is processed, and the serum is separated from the clot.

b. Hemoglobin was measured at the sentinel sites using multianalizators.

c. Iron deficiency in survey participants has been measured using ferritin concentration in serum. Ferritin was tested in serum using the ELISA method.

d. Folate deficiency has been measured using serum folate concentration measured on serum separated from blood. Testing was done using an ELISA method and a microbiologic test kit (DRG International Inc., USA. BIO-4886).

\section{Results}

The study in pregnant women showed $14.4 \%$ prevalence of anemia in 3030 pregnant enrolling in study; the percentage of iron deficiency in total (963 pregnant with lab. Testing of blood on ferritin) resulted in 57\%. And 28\% of folate deficiency in 963 pregnant (with lab. testing on serum folate) (Figures 1\&2). The study in children (12-23 months of age) showed about $36 \%$ prevalence of anemia in 1179 children enrolling in study; the percentage of iron deficiency in total (1021 children with lab. Testing of blood on ferritin) resulted in $74 \%$. Severe anemia cases were not identified in both groups, not in toddlers and not in pregnant women (Figure 3). Regarding regional profiles, we have detected that prevalence in toddlers in Samegrelo region (24.2\%) anemia percentage is 1.5 - 2 times less than in other three regions, especially when comparing 
with Achara (47.0\%) (Table 1). Reviewing regional profiles for Iron deficiency, showed that prevalence in studied 4 regions is almost the same, but with the high prevalence. (Table 2). High prevalence and a big difference in folate deficiency were identified between
EAST and WEST parts of Georgia (21-24\% in Tbilisi\&Kakhetivs 34.4-37\% in Achara\&Samegrelo) / (Table 3). Surveillance system revealed high rate of NTDs prevalence (it's about 4 per 1000 livebirth) (Table 4).

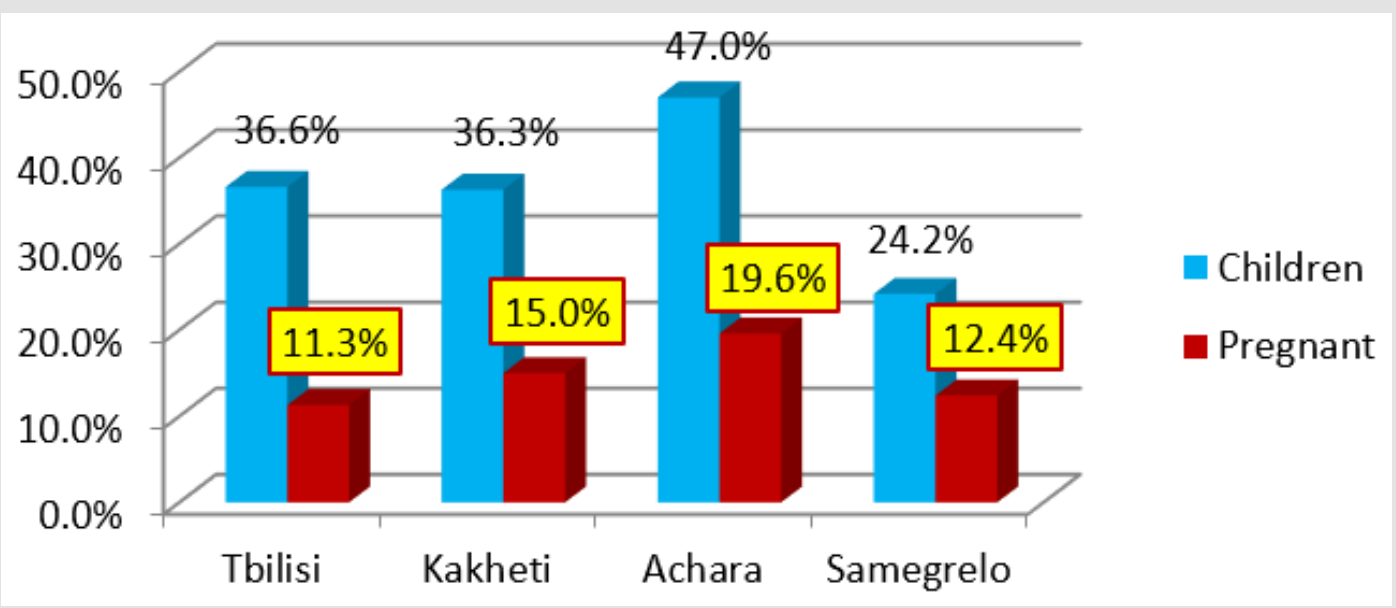

Figure 1: Anemia prevalence (percentage) by regions and target groups.

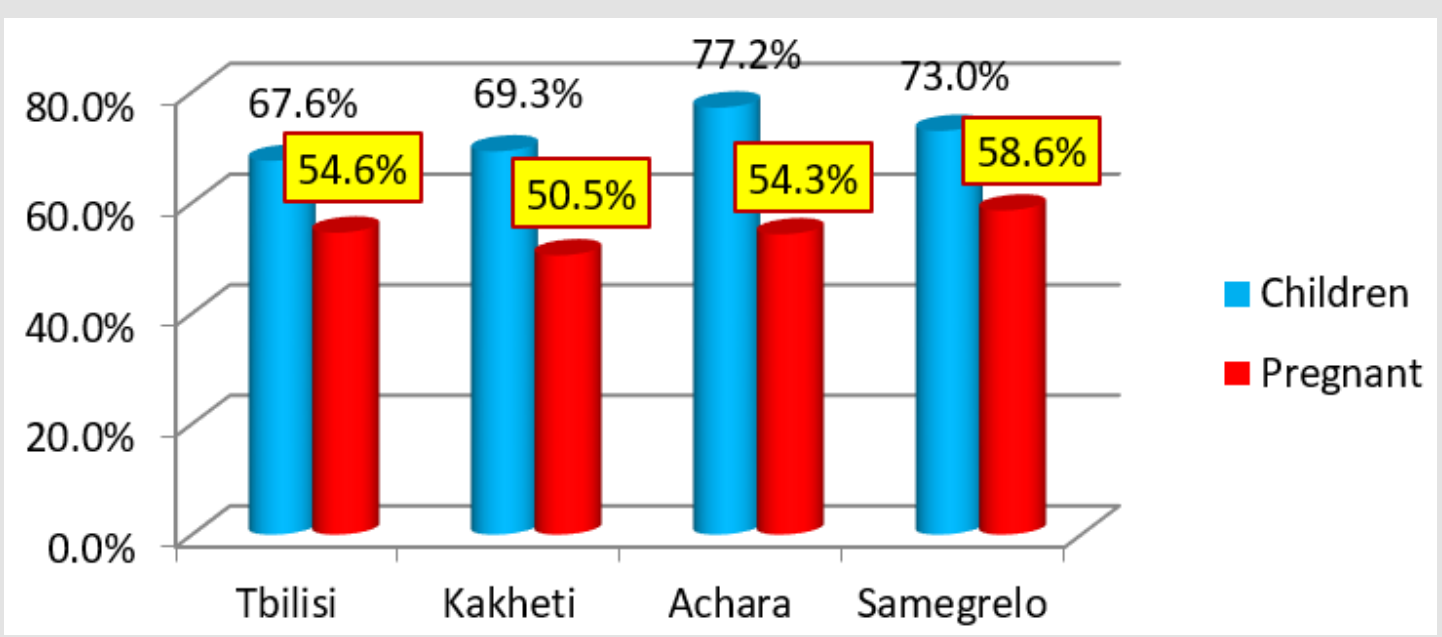

Figure 2: Iron deficiency prevalence (percentage) by regions and target groups.

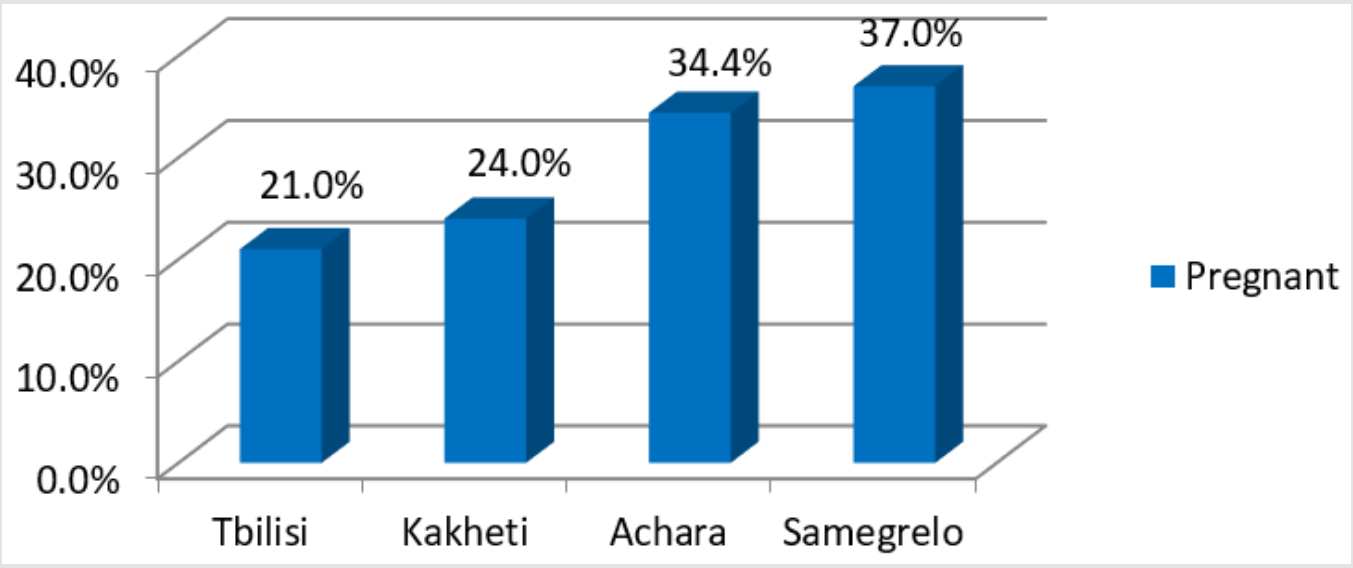

Figure 3: Folate deficiency prevalence (percentage) by regions and target group. 
Table 1: Hemoglobin (anemia?) /2016-2019.

\begin{tabular}{|c|c|c|c|c|c|c|}
\hline Years & $\begin{array}{c}\mathbf{1 2 - 2 3} \text { moths age } \\
\text { children }\end{array}$ & $\begin{array}{c}\text { Anemia } \\
\mathbf{H b}<\mathbf{1 1 0} \mathbf{g} / \mathbf{l}\end{array}$ & $\begin{array}{c}\text { Severe anemia } \\
\mathbf{H b}<\mathbf{7 0} \mathbf{g} / \mathbf{l}\end{array}$ & Pregnant (1 $\mathbf{1}^{\text {st } \text { trimester) }}$ & $\begin{array}{c}\text { Anemia } \\
\mathbf{H b}<\mathbf{1 1 0} \mathbf{g} / \mathbf{l}\end{array}$ & $\begin{array}{c}\text { Anemia } \\
\mathbf{H b}<\mathbf{7 0} \mathbf{g} / \mathbf{l}\end{array}$ \\
\hline & & & & & \\
\hline total & 1179 & $36.2 \%$ & $0.0 \%$ & 3030 & $14.4 \%$ & $0.0 \%$ \\
\hline
\end{tabular}

Table 2: Ferritin (Iron)Deficiency.

\begin{tabular}{|c|c|c|c|c|}
\hline Years & $\mathbf{1 2 - 2 3}$ moths age children & Ferritin $<\mathbf{1 2 . 0} \mathbf{\mu g} / \mathbf{L}$ & pregnant (1 ${ }^{\text {st }}$ trimester) & Ferritin < 15.0 $\mathbf{\mu g} / \mathbf{L}$ \\
\hline 2016 & 238 & $84.4 \%$ & 243 & $67.4 \%$ \\
\hline 2017 & 240 & $83.3 \%$ & 240 & $54.6 \%$ \\
\hline 2018 & 303 & $74 \%$ & 240 & $57.2 \%$ \\
\hline 2019 & 240 & $56 \%$ & 240 & $47 \%$ \\
\hline total & 1021 & $74 \%$ & 963 & $57 \%$ \\
\hline
\end{tabular}

Table 3: Folate Deficiency.

\begin{tabular}{|c|c|c|}
\hline Years & Pregnant $\left(\mathbf{1}^{\text {st }}\right.$ trimester) & (Folate Deficiency) Serum Folate $<\mathbf{3 . 0} \mathbf{n g} / \mathbf{m l}$ \\
\hline 2016 & 243 & $31 \%$ \\
\hline 2017 & 240 & $23 \%$ \\
\hline 2018 & 240 & $30 \%$ \\
\hline 2019 & 240 & $29 \%$ \\
\hline total & $\mathbf{9 6 3}$ & $\mathbf{2 8 \%}$ \\
\hline
\end{tabular}

Table 4: NTDs prevalence /2016-2019/data is obtained from sentinels, only.

\begin{tabular}{|c|c|c|c|}
\hline Years & Live births & NTDs (Neural tube defects) & NTDs per 1000 live birth \\
\hline 2016 & 2910 & 8 & 2.75 \\
\hline 2017 & 2969 & 8 & 6.69 \\
\hline 2018 & 2862 & 16 & 3.80 \\
\hline 2019 & 3156 & 12 & \\
\hline & & & 3.7 \\
\hline
\end{tabular}

\section{Discussion \& Conclusion}

Due to similar studies in similar context, we can assume that above mentioned deficiencies are caused due to possible reasons Georgian foods do not contain enough micronutrients and women of reproductive age have not adequate information about folate deficiency and its impact to NTDs development. After reviewing of analyses of the sentinel approach surveillance system's 4 years (2016-2019 yy) functioning results we can conclude that: we have Folate deficiency problem in pregnant; Iron deficiency problem, that is reflection of the general situation existing in Georgia. According to expert's opinion it is the quite enough evidences for conclusion and recommendations for the initiation of nutrition interventions (mainly, food fortification) and for making the adequate changes/ amendments in relative legislation.

\section{Recommendations}

- Need to advocate for nutrition interventions regarding food fortification (with iron and folic acid) strategy.
- Promote the main principle of healthy eating. And receiving of folic acid supplements.

\section{Acknowledgement}

This research study was funded by the United States Centers for Disease Control and Prevention (Strengthening micronutrients deficiency surveillance in Georgia- grant \# 5U2GGH001658). The research team/NCDC acknowledges with gratitude the commitment of the US CDC to the research efforts, Deputy NCDC General Director (P. Imnadze), and General Director of NCDC Georgia (A. Gamkrelidze). Also, Staff of sentinel sites (Health Facilities) and Laboratories involved in the project.

\section{References}

1. Bailey R L, West Jr K P b, Black R E b (2015) The Epidemiology of Global Micronutrient Deficiencies. Ann Nutr Metab 2: 22-33

2. (2000) Armenia Demographic and Health Survey.

3. GNS $-2009 /$ report book/Georgia-UNICEF 
4. Iron Deficiency Anaemia Assessment, Prevention and Control/ A guide for programme managers.

5. De Benoist B (2008) Conclusions of a WHO Technical Consultation on folate and vitamin B12 deficiencies. Food and Nutrition Bulletin 29(2): 238-244.

6. Katie A Burren, Dawn Savery, Valentina Massa, Robert M Kok, John M Scott, et al. (2008) Gene-environment interactions in the causation of neural tube defects: folate deficiency increases susceptibility conferred by loss of Pax3 function. Human Molecular Genetics 17(23): 3675-3685

7. J Safi, L Joyeux, G E Chalouhi (2012) Periconceptional Folate Deficiency and Implications in Neural Tube Defects. Journal of Pregnancy pp. 295083

ISSN: 2574-1241

DOI: $10.26717 /$ BJSTR.2019.23.003853

Robizon Tsiklauri. Biomed J Sci \& Tech Res

(c) (P) This work is licensed under Creative

Submission Link: https://biomedres.us/submit-manuscript.php
8. Ayaz Reyhan, Asoglu, Mehmet Resit (2019) Is low folate status or vitamin B12 deficiency or both associated with a high rate of NTDs. The Journal of Maternal-Fetal\& Neonatal Medicine Neural tube defects in eastern Turkey 1-6.

9. Haochen Wang, Hans De Steur (2016) Effectiveness of Folic Acid Fortified Flour for Prevention of Neural Tube Defects in a High-Risk Region. Nutrients 8(3): 152.

10. Mauren M Black (2008) Effects of Vitamin B12 and Folate Deficiency on Brain Development in Children. Food and Nutrition Bulletin 29(2): 126131.

$\begin{array}{ll}\text { BIOMEDICAL } & \text { Assets of Publishing with us } \\ \text { RESEARCHES } & \text { - Global archiving of articles } \\ & \text { - Immediate, unrestricted online access } \\ & \text { - Rigorous Peer Review Process } \\ \end{array}$

